\title{
Comparaison de la radiosensibilité de la variété xanthi Dulieu du tabac (Nicotiana tabacum L.) à celle de la variété dont elle dérive ${ }^{(1)}$
}

\author{
C. ALMHANA*, M. DELPOUX ${ }^{\star \star}$, J.-P. LACOMBE ${ }^{\star \star \star}$ \\ (Manuscrit reçu le 5 juin 1987)
}

\begin{abstract}
RÉSUMÉ
Des graines de deux variétés de Nicotiana tabacum (var. xanthi et xanthi Dulieu) ont été irradiées (rayonnement $\gamma$ ) à des doses croissantes $(100,500$, 800,1000 et $1500 \mathrm{~Gy}$ ) et les effets des irradiations ont été observés pour différents paramètres relatifs à la germination et à la morphologie des plantules. Les graines des deux variétés montrent (i) une grande radiorésistance, (ii) sauf exception (pourcentage de plantules ayant formé deux cotylédons significativement plus faibles chez la variété xanthi), la différence de sensibilité entre les deux variétés est peu importante; toutefois, il semble que la variété xanthi Dulieu soit légèrement plus radiorésistante, (iii) une réponse dose-effet du même type quels que soient la variété et le critère biologique retenus: une stimulation à faible dose du gradient choisi; une inhibition de la germination et du développement aux plus fortes doses (1 000-1 500 Gy).
\end{abstract}

\section{ABSTRACT}

Seeds of two varieties of Nicotiana tabacum (Var. xanthi and xanthi Dulieu) were exposed to increasing doses of $\gamma$ radiations $(100,500,800,1000,1500$ Gy). The irradiation effects were observed for several parameters relating to germination and plantlet morphology.

The seeds of these two varieties showed (i) a high radioresistance, (ii) except for the number of seedlings able to grow until the two-cotyledon stage which is significantly lower for var. xanthi, there was a very slight difference between the two varieties; however, it seems that var. xanthi Dulieu was a little more resistant, (iii) the dose-effect relationship was similar in both varieties, whatever the biological criterion chosen: stimulation at the lowest dose (100 Gy); inhibition of germination and development at the highest doses (1 000-1500 Gy).

(1) Ce travail fait partie d'une thèse consacrée à l'étude de l'action des rayonnements ionisants (faibles et fortes doses), sur les graines, plantules et parties reproductrices mâles de deux variétés de tabac.

* Ecole nationale supérieure agronomique de Toulouse (ENSAT), Laboratoire de cytologie et pathologie végétale, 145, avenue de Muret, 31076 Toulouse Cedex.

** Université Paul-Sabatier, Laboratoire de botanique et de biogéographie, 39, allée Jules Guesde, 31000 Toulouse Cedex.

*** Ecole nationale supérieure agronomique de Toulouse, Laboratoire d'informatique appliquée, 145, avenue de Muret, 31076 Toulouse Cedex. 


\section{INTRODUCTION}

Plusieurs travaux ont été réalisés en soumettant la variété xanthi Dulieu du tabac (Nicotiana tabacum L.) à de faibles et très faibles doses chroniques d'irradiation gamma $[3-4,15]$ ainsi qu'à l'action d'autres facteurs de l'environnement: pollution atmosphérique [6-8], composition chimique des substrats [3-4].

Sous irradiation chronique, les débits de doses utilisés étaient compris entre le bruit de fond naturel $\left(0,02 \times 10^{-3} \mathrm{cGy} / \mathrm{h}\right.$, soit $\left.175 \times 10^{-3} \mathrm{cGy} / \mathrm{an}\right)$ et $10^{-2} \mathrm{~Gy} / \mathrm{h}$, soit $87,6 \mathrm{~Gy} / \mathrm{an}$. Dans les différentes expériences, aucun plant utilisé n'est mort, ce qui démontre que les doses délivrées étaient inférieures aux doses létales. Or, des changements significatifs de la structure du marqueur génétique ont été observés sur les plants soumis à des doses caractérisées par des débits égaux ou supérieurs à $0,3-0,4 \times 10^{-3} \mathrm{cGy} / \mathrm{h}$ soit 2,6 à 3,5 cGy/an. Au-dessus de cette valeur seuil, une relation dose-effet linéaire a été mise en évidence $[4-5,15]$.

Pour cela, les effets de l'irradiation (0 à $1500 \mathrm{~Gy}$ ) de graines des deux variétés ont été observés et quantifiés pour la germination (déclenchement, durée pour atteindre le maximum de germination, taux de germination, énergie germinative), la faculté germinative et la morphologie des constituants des plantules: racines, hypocotyle, cotylédons.

\section{MATÉRIEL ET MÉTHODES}

\section{II.1. Matériel}

Les deux variétés de tabac expérimentées sont: (i) la variété xanthi de Nicotiana tabacum L., caractérisée par une synthèse chlorophyllienne normale et, de ce fait, de couleur vert-foncé, (ii) la variété vert-jaune obtenue par DULIEU [10-14] à partir de la première en la soumettant à l'action du MSE (méthane sulfonate d'éthyle). Sa déficience chlorophyllienne partielle lui donne une couleur vert-jaune.

L'irradiation a été appliquée à des graines des deux variétés ayant un pourcentage d'humidité de $4 \%$ à l'aide d'un appareil du type "Gammacell" débitant $200 \mathrm{~Gy} / \mathrm{h}$; les graines avaient été préalablement introduites dans de petits tubes en matière plastique.

\section{II.2. Méthodes}

\section{II.2.1. Germination et culture des plantules}

Immédiatement après l'irradiation les graines sont semées sur milieu gélosé stérilisé placé au fond de boîtes en matière plastique transparente avec les dimensions suivantes: $35 \times 23,5 \times 13,5 \mathrm{~cm}$. Les boîtes sont stérilisées et contiennent chacune 1,5 litre de milieu gélosé de MURASCHIGE et SKOOG [22] modifié par LINSHAIER et SKOOG [19]. Le pH a été ajusté à 5,6 (optimum de développement pour les plantules) par addition de 
quelques gouttes de solution de $\mathrm{NaOH}$ normale. Dans une première série d'expériences destinées à évaluer les effets de l'irradiation sur la germination et la faculté germinative, on trace des traits sur le fond de chaque boîte, divisant ainsi le milieu de culture en six parties égales. Chaque partie est ensemencée par un lot de $\mathbf{3 0}$ graines, chaque lot ayant reçu l'une des six doses choisies: 0 (témoin), 100, 500, 800, 1000,1500 Gy. Après ensemencement en conditions stériles, les boîtes sont fermées par un couvercle et placées dans une chambre climatisée à la température de $28 \pm 1^{\circ} \mathrm{C}$ le jour, de $25 \pm 1^{\circ} \mathrm{C}$ la nuit. La photopériode est de $12 \mathrm{~h} / 12 \mathrm{~h}$, l'intensité lumineuse de $5000 \mathrm{lux} / \mathrm{m}^{2}$.

\section{II.2.2. Critères biologiques}

- Germination (fig. 1):

- Déclenchement de la germination: c'est le temps (temps de latence) compris entre la date de semis et la date d'apparition des premiers cotylédons $=$ a.

- Durée nécessaire pour atteindre le pourcentage maximal de germination: elle correspond au temps séparant la première germination de la dernière $=\mathrm{b}$.

- Taux de germination: il correspond au pourcentage maximal de graines ayant produit des plantules à deux cotylédons $=\mathrm{c}$.

- Energie germinative: c'est le temps qui sépare la germination de la première graine de la germination de $50 \%$ des graines $=\mathrm{e}$.

- Faculté germinative et taux de mortalité: la faculté germinative est le nombre pour cent de plantes bien constituées mesuré 30 jours après le semis par rapport au nombre de plantules à deux cotylédons constituant le lot de départ. Le pourcentage de plantules initiales ayant dégénéré représente le taux de mortalité, les conditions de culture étant considérées comme les meilleures possibles [23].

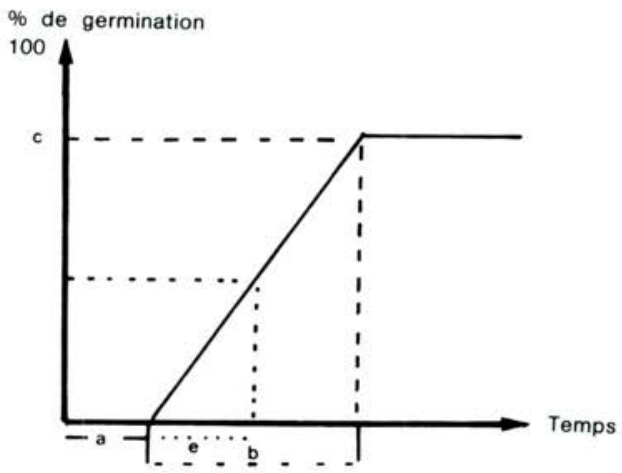

Fig. 1. - Critères retenus pour évaluer l'influence de l'irradiation sur la germination.

$\mathrm{a}=$ déclenchement de la germination, $\mathrm{b}=$ durée nécessaire pour atteindre le $\%$ maximal de germination, $\mathrm{c}=$ taux de germination, $\mathrm{e}=$ énergie germinative. 
- Morphologie des plantules:

- Observations 10 jours après la germination

a) Nombre de racines,

b) Longueur totale de la plantule,

c) Longueur de l'hypocotyle,

d) Surface des cotylédons;

- Observations 30 jours après la germination

a) Longueur de la racine principale,

b) Longueur de I'hypocotyle,

c) Longueur de la tige (mesurée de l'hypocotyle à la base de la dernière feuille individualisée).

\section{II.2.3. Méthodes statistiques}

Tous les tests d'hypothèses ont été exécutés avec un risque de première espèce de $5 \%$.

Pour chaque variable étudiée, on a effectué une analyse de variance selon un plan factoriel:

facteur $A$ : variétés de tabac ( 2 niveaux),

facteur B: doses de rayons gamma (6 niveaux),

nombre de répétitions: 3 .

On a utilisé le modèle I (à effets fixés).

L'examen des résidus n'a pas révélé de valeurs suspectes.

Chaque fois que le test $F$ s'est avéré significatif, nous avons effectué une comparaison multiple des moyennes à l'aide du test de NEWMAN et KEULS. Nous présentons, dans les tableaux ci-après, les moyennes, par variable, assorties de leurs intervalles de confiance. L'affectation des moyennes à un groupe homogène est figurée par une lettre $a, b, c$, etc. II y a autant de groupes statistiquement homogènes qu'il y a de lettres. Dans tous les cas les valeurs discutées correspondent aux moyennes de trois répétitions.

\section{RÉSULTATS}

\section{III.1. Effet sur la germination}

- Déclenchement de la germination

Variété xanthi (fig. 2a): la germination commence 5 jours après le semis chez le lot témoin. Chez les lots irradiés, le lot 100 Gy présente une certaine "précocité" par rapport au témoin puisque les premières germinations apparaissent le $4^{\mathrm{e}}$ jour. Par contre, le lot 500 Gy est retardé d'un jour et ceux de 800,1000 et 1500 Gy sont uniformément retardés de 2 jours.

Variété xanthi Dulieu (fig. 2b): on observe une certaine similarité avec la variété xanthi en ce qui concerne la "précocité" du lot 100 Gy (4, 2 j.); par contre, les autres lots (témoin et traités), germent presque simultanément ( $5^{\mathrm{e}}$ jour). 
RADIOSENSIBILITÉ DE LA VARIÉtÉ XANTHI DULIEU DU TABAC (NICOTIANA TABACUM L)

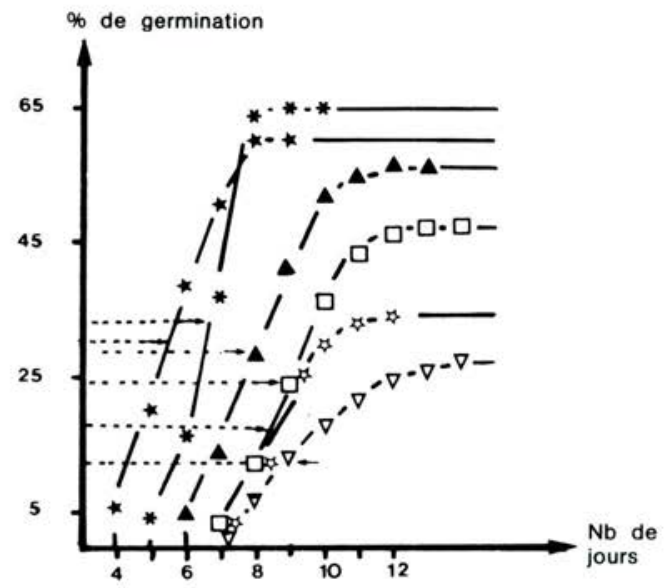

Fig. 2a. - Influence de différentes doses de rayonnement $\gamma$ sur la germination de la variété xanthi.

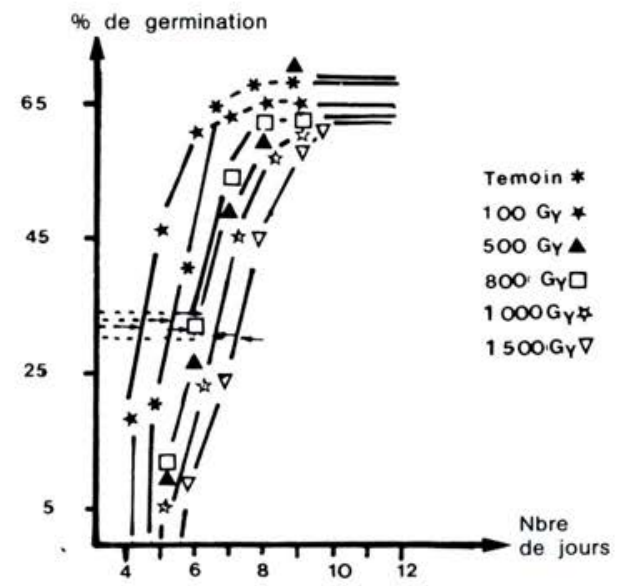

Fig. 2b. - Influence de différentes doses de rayonnement $\gamma$ sur la germination de la variété xanthi Dulieu.

- Durée nécessaire pour atteindre le pourcentage maximal de germination

Chez la variété xanthi (fig. 2a), cette durée augmente régulièrement du témoin à 500 Gy; elle est de 3,5 et 7 jours respectivement pour le témoin, 100 et 500 Gy, puis elle se stabilise à 7 jours pour les traitements supérieurs.

Chez la variété xanthi Dulieu (fig. $2 b$ ), la réaction est différente: la durée est égale à 4 jours, quelle que soit la dose.

VOL. $23-\mathrm{N}^{\circ} 1$ 


\section{- Taux de germination}

Chez la variété xanthi (tabl. I et fig. 3), le taux de germination, qui est d'environ $65 \%$ chez le témoin, diminue régulièrement en raison inverse de la dose d'irradiation et atteint $26,5 \%$ pour 1500 Gy; cependant, la chute du taux de germination par rapport au témoin n'est statistiquement significative qu'au-delà de 800 Gy.

Chez la variété xanthi Dulieu (tabl. I et fig. 3), le taux de germination, qui est de $68,5 \%$ chez le témoin, n'est modifié de façon significative par aucun des traitements. Cette variété semble donc plus radiorésistante que l'autre.

TABLEAU I

Influence de différentes doses de rayonnement $\gamma$ (en Gy) sur le taux de germination de deux variétés de tabac

\begin{tabular}{|c|c|c|c|c|c|c|c|}
\hline \multirow{4}{*}{ Dulieu } & Doses & Témoin & 100 & 500 & 800 & 1000 & 1500 \\
\cline { 2 - 8 } & $\%$ & $\begin{array}{c}68,43+ \\
1,35\end{array}$ & $\begin{array}{c}66,43+ \\
0,66\end{array}$ & $\begin{array}{c}68,77+ \\
1,30\end{array}$ & $\begin{array}{c}64,60+ \\
1,30\end{array}$ & $\begin{array}{c}63,90+ \\
0,93\end{array}$ & $\begin{array}{c}63,10+ \\
0,86\end{array}$ \\
\cline { 2 - 8 } & Signif. & $\mathrm{c}$ & $\mathrm{c}$ & $\mathrm{c}$ & $\mathrm{c}$ & $\mathrm{c}$ & $\mathrm{c}$ \\
\hline \multirow{4}{*}{ xanthi } & $\%$ & $\begin{array}{c}64,60+ \\
0,23\end{array}$ & $\begin{array}{c}60,30+ \\
0,55\end{array}$ & $\begin{array}{c}57,10+ \\
1,10\end{array}$ & $\begin{array}{c}47,60+ \\
0,93\end{array}$ & $\begin{array}{c}33,70+ \\
0,66\end{array}$ & $\begin{array}{c}26,50+ \\
0,14\end{array}$ \\
\cline { 2 - 8 } & Signif. & $\mathrm{c}$ & $\mathrm{c}$ & $\mathrm{c}$ & $\mathrm{b}$ & $\mathrm{a}$ & $\mathrm{a}$ \\
\hline
\end{tabular}

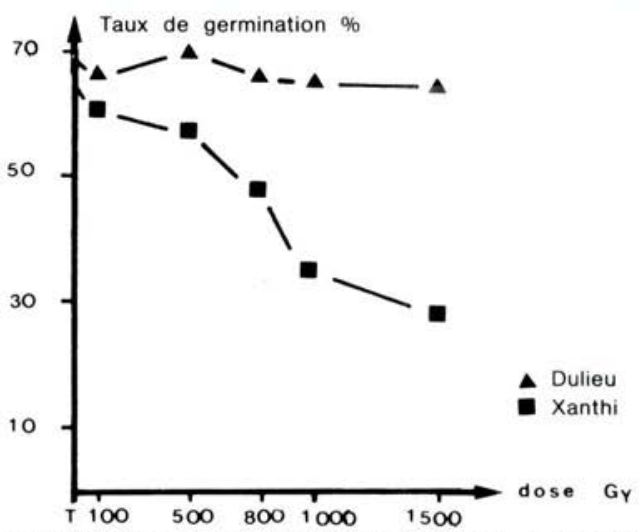

Fig. 3. - Influence de différentes doses de rayonnement $\gamma$ sur le taux de germination des deux variétés de tabac.

\section{- Energie germinative}

Les énergies germinatives sont nettement différentes chez les deux variétés. Cependant, dans les deux cas, celles relatives au témoin et à $100 \mathrm{~Gy}$ sont sensiblement équivalentes.

Elles diminuent assez nettement à partir de 500 Gy; l'analyse de variance n'a pas révélé d'interaction significative (voir tableau II). Notons cependant que les énergies germinatives déterminées pour 1500 Gy sont inférieures à celles des témoins de $50 \%$ pour la variété xanthi Dulieu et de $100 \%$ pour la variété xanthi (fig. $2 a$ et $2 \mathrm{~b}$ ). 
RADIOSENSIBILITE DE LA VARIÉtÉ XANTHI DULIEU DU TABAC (NICOTIANA TABACUM L)

TABLEAU ॥

Influence de différentes doses de rayonnement $\gamma$ (en Gy) sur l'énergie germinative de deux variétés de tabac

\begin{tabular}{|c|c|c|c|c|c|c|c|}
\hline \multirow{4}{*}{ Dulieu } & Doses & Témoin & 100 & 500 & 800 & 1000 & 1500 \\
\cline { 2 - 8 } & $\begin{array}{c}\text { Nbre de } \\
\text { jours }\end{array}$ & $\begin{array}{c}1,53+ \\
0,01\end{array}$ & $\begin{array}{c}1,50+ \\
0\end{array}$ & $\begin{array}{c}2,00+ \\
0,04^{+}\end{array}$ & $\begin{array}{c}2,00+ \\
0\end{array}$ & $\begin{array}{c}2,00+ \\
0\end{array}$ & $\begin{array}{c}2,33+ \\
0,06\end{array}$ \\
\cline { 2 - 8 } & Signif. & $\mathrm{a}$ & $\mathrm{a}$ & $\mathrm{a}$ & $\mathrm{a}$ & $\mathrm{a}$ & $\mathrm{a}$ \\
\hline \multirow{4}{*}{ xanthi } & $\begin{array}{c}\text { Nbre de de } \\
\text { jours }\end{array}$ & $\begin{array}{c}0,67+ \\
0,03\end{array}$ & $\begin{array}{c}0,63+ \\
0,13^{+}\end{array}$ & $\begin{array}{c}1,37+ \\
0,07^{+}\end{array}$ & $\begin{array}{c}1,17+ \\
0,08\end{array}$ & $\begin{array}{c}1,27+ \\
0,01\end{array}$ & $\begin{array}{c}1,33+ \\
0,06\end{array}$ \\
\cline { 2 - 8 } & Signif. & $\mathrm{a}$ & $\mathrm{a}$ & $\mathrm{a}$ & $\mathrm{a}$ & $\mathrm{a}$ & $\mathrm{a}$ \\
\hline
\end{tabular}

\section{III.2. Effets de l'irradiation sur la faculté germinative et le taux de mortalité}

Les facultés germinatives des deux variétés (tableau III et fig. 4), qui sont proches de $100 \%$ pour le témoin et le traitement $100 \mathrm{~Gy}$, diminuent nettement en raison inverse de la dose, ce de façon similaire chez les deux variétés. On note que le taux de mortalité (tableau III) des jeunes plantules augmente un peu plus rapidement chez la variété xanthi pour les irradiations supérieures à 800 Gy. Toutes les plantules issues de graines irradiées à la dose de 1500 Gy meurent dans les 30 jours suivant le semis.

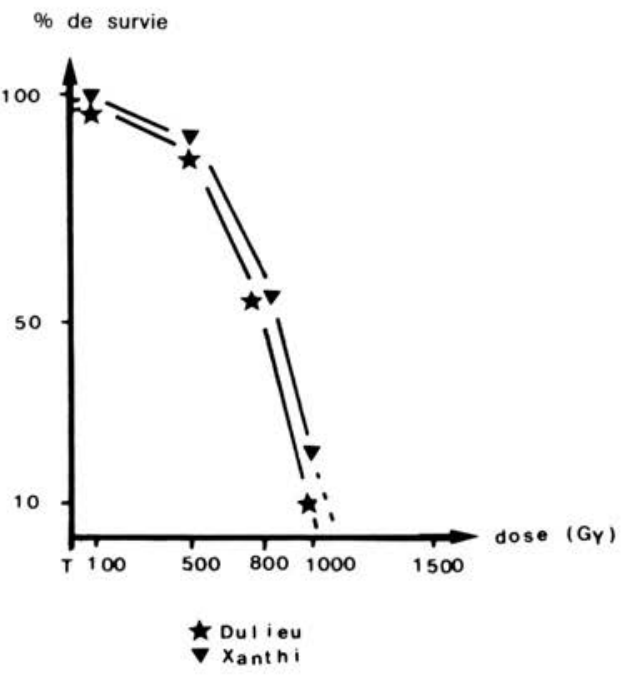

Fig. 4. - Influence de différentes doses de rayonnement $\gamma$ sur la faculté germinative chez deux variétés de tabac, 30 Jours après le semis. 
TABLEAU III

\begin{abstract}
Influence de différentes doses de rayonnement $\gamma$ (en Gy) sur le pourcentage de mortalité de deux variétés de tabac déterminé 30 jours après le semis.
\end{abstract}

\begin{tabular}{|c|c|c|c|c|c|c|c|}
\hline \multirow{4}{*}{ Dulieu } & Doses & Témoin & 100 & 500 & 800 & 1000 & 1500 \\
\cline { 2 - 8 } & $\%$ & $\begin{array}{c}0,70+ \\
0,1\end{array}$ & $\begin{array}{c}0,95+ \\
0,03\end{array}$ & $\begin{array}{c}10,91+ \\
0,3\end{array}$ & $\begin{array}{c}45,9+ \\
0,52\end{array}$ & $\begin{array}{c}80,2+ \\
0,3\end{array}$ & $\begin{array}{c}100+ \\
0\end{array}$ \\
\cline { 2 - 8 } & Signif. & $\mathrm{a}$ & $\mathrm{a}$ & $\mathrm{b}$ & $\mathrm{c}$ & $\mathrm{d}$ & $\mathrm{f}$ \\
\hline \multirow{3}{*}{ xanthi } & $\%$ & $\begin{array}{c}0,49+ \\
0,07\end{array}$ & $\begin{array}{c}1,66+ \\
0,14\end{array}$ & $\begin{array}{c}12,2+ \\
0,12\end{array}$ & $\begin{array}{c}45,3+ \\
0,43\end{array}$ & $\begin{array}{c}91,6+ \\
0,22\end{array}$ & $\begin{array}{c}100+ \\
0\end{array}$ \\
\cline { 2 - 8 } & Signif. & $\mathrm{a}$ & $\mathrm{a}$ & $\mathrm{b}$ & $\mathrm{c}$ & $\mathrm{e}$ & $\mathrm{f}$ \\
\hline
\end{tabular}

III.3. Effets de l'irradiation sur la morphogenèse des plantules issues de graines irradiées

\title{
III.3.1. Observations effectuées 10 jours après la germination
}

Une plantule témoin de tabac âgée de 10 jours a (i) un système radiculaire formé, avec une racine principale bien développée (de l'ordre de $0,90 \mathrm{~cm}$ ) et quelques racines secondaires latérales (3-4 selon la variété), (ii) un hypocotyle dont l'élongation atteint environ $0,45 \mathrm{~cm}$ et les deux cotylédons bien étalés.

\section{- Nombre de racines}

Pour les deux variétés (tableau IV et fig. 5), la réponse aux doses croissantes est sensiblement de même type: à $100 \mathrm{~Gy}$, la rhizogenèse est presque identique à celle du témoin (var. xanthi Dulieu) ou même légèrement stimulée (var. xanthi), mais les irradiations supérieures réduisent progressivement et significativement le nombre de racines formées qui atteint un palier à 1000 Gy correspondant à l'émission d'un seul élément radiculaire. L'activité rhizogène de la variété xanthi, bien que stimulée à $100 \mathrm{~Gy}$, est plus sensible à l'irradiation; en effet, chez les témoins, le nombre de racines de cette variété $(4,63+0,07)$ est initialement supérieur à celui de la variété xanthi Dulieu $(3,70+0,06)$.

\section{TABLEAU IV}

Influence de différentes doses de rayonnement $\gamma$ (en Gy) sur le nombre de racines émises par deux variétés de tabac, mesuré 10 jours après le semis

\begin{tabular}{|c|c|c|c|c|c|c|c|c|}
\hline \multirow{3}{*}{ Dulieu } & Doses & Témoin & 100 & 300 & 500 & 800 & 1000 & 1500 \\
\hline & $\begin{array}{l}\text { Nbre de } \\
\text { racines }\end{array}$ & $\begin{array}{c}3,70 \\
0,06\end{array}+$ & $\underset{0,04}{3,50}+$ & ${ }_{0,12}^{4,70}+$ & ${ }_{0,03}^{1,47}+$ & $\begin{array}{c}1,10 \\
0,04\end{array}+$ & $\begin{array}{c}1,03 \\
0,01\end{array}+$ & $\begin{array}{c}1,00 \\
0\end{array}+$ \\
\hline & Signif. & c & c & d & $a b$ & a & a & a \\
\hline \multirow{2}{*}{ xanthi } & $\begin{array}{c}\text { Nbre de } \\
\text { racines }\end{array}$ & $\begin{array}{c}4,63 \\
0,07\end{array}$ & $\begin{array}{c}5,67 \\
0,09\end{array}+$ & $\begin{array}{c}5,87 \\
0,10\end{array}+$ & $\begin{array}{c}3,20 \\
0,04\end{array}+$ & $\begin{array}{c}1,83 \\
0,05\end{array}+$ & $\begin{array}{c}1,03 \\
0,01\end{array}+$ & $\begin{array}{c}1,03 \\
0,01\end{array}+$ \\
\hline & Signif. & d & e & e & c & b & a & a \\
\hline
\end{tabular}




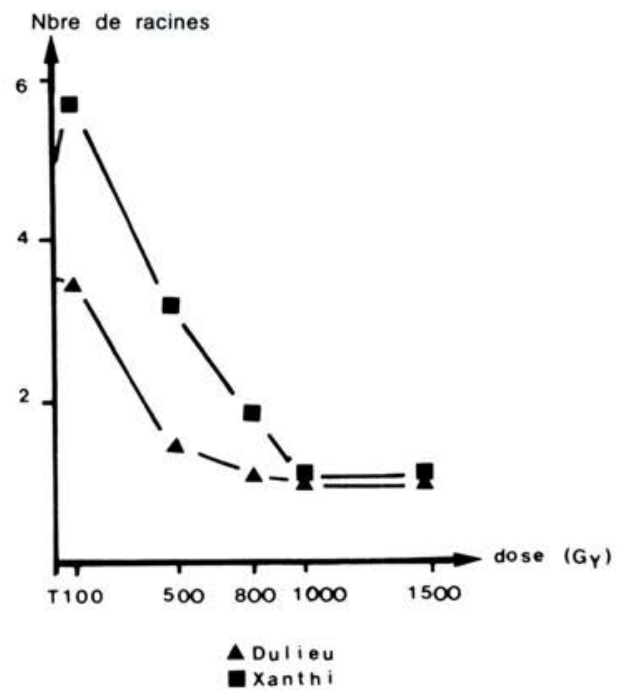

Fig. 5. - Influence de différentes doses de rayonnement $\gamma$ sur le nombre de racines émises par deux variétés de tabac, 10 jours après le semis.

\section{- Longueur de la racine principale}

L'irradiation à 100 Gy des graines ne modifie pas le développement de la racine principale au cours des 10 premiers jours après le semis; tout au plus peut-on noter une très faible stimulation (non significative) de son allongement. Par contre, pour les irradiations supérieures à 100 Gy, le développement radiculaire subit une inhibition qui, à 1000 Gy, atteint $90 \%$ (tabl. IV et fig. 6).

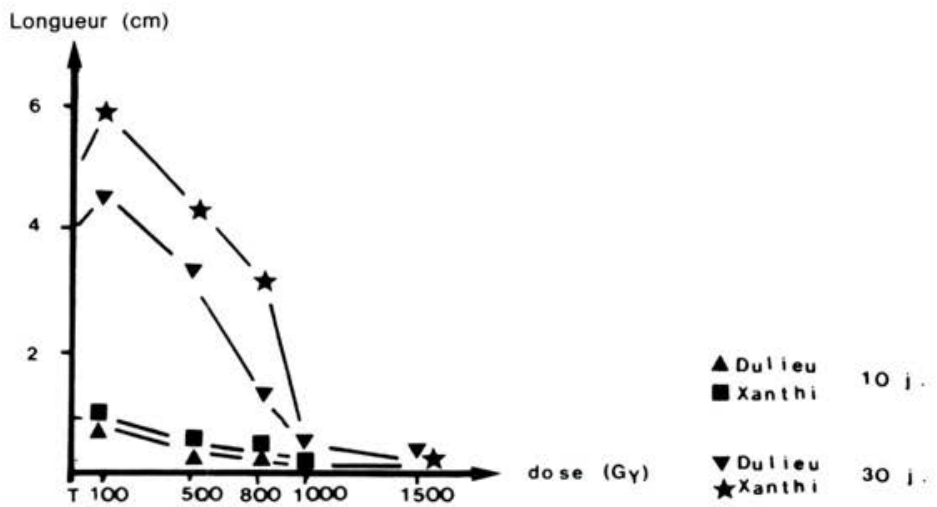

Fig. 6. - Influence de différentes doses de rayonnement $\gamma$ sur la longueur de la racine mesurée chez deux variétés de tabac, 10 et 30 jours après le semis. 


\section{- Longueur de l'hypocotyle}

Dix jours après le semis, l'allongement de l'hypocotyle chez les deux variétés est plus important pour la dose de 100 Gy, équivalent à celui des témoins pour la dose 500 Gy et réduit pour les doses supérieures (réduction de $50 \%$ (var. xanthi Dulieu) à $60 \%$ (var. xanthi) par rapport aux témoins pour les irradiations de $1000 \mathrm{~Gy}$ ). Cependant, le test statistique ne donne aucune signification aux variations inter ou intravariétales mesurées (tableau $V$ et fig. 7).

Les deux variétés ne présentent pas de différence significative.

\section{TABLEAU $V$}

Influence de différentes doses de rayonnement $\gamma$ (en Gy)

sur la longueur de l'hypocotyle mesurée chez deux variétés de tabac, 10 et 30 jours après le semis

10 jours

\begin{tabular}{|c|c|c|c|c|c|c|c|}
\hline \multirow{4}{*}{ Dulieu } & Doses & Témoin & 100 & 500 & 800 & 1000 & 1500 \\
\cline { 2 - 8 } & $\begin{array}{c}\text { Long. de } \\
\text { I'hypoc. }\end{array}$ & $\begin{array}{c}0,46+ \\
0,024\end{array}$ & $\begin{array}{c}0,51+ \\
0,022\end{array}$ & $\begin{array}{c}0,46+ \\
0,018^{+}\end{array}$ & $\begin{array}{c}0,33+ \\
0,11\end{array}$ & $\begin{array}{c}0,25+ \\
0,006\end{array}$ & $\begin{array}{c}0,22+ \\
0,006\end{array}$ \\
\cline { 2 - 8 } & Signif. & $\mathrm{a}$ & $\mathrm{a}$ & $\mathrm{a}$ & $\mathrm{a}$ & $\mathrm{a}$ & $\mathrm{a}$ \\
\hline \multirow{5}{*}{ xanthi } & $\begin{array}{c}\text { Long. de } \\
\text { I'hypoc. }\end{array}$ & $\begin{array}{c}0,43+ \\
0,006\end{array}$ & $\begin{array}{c}0,52+ \\
0,002\end{array}$ & $\begin{array}{c}0,43+ \\
0,008^{+}\end{array}$ & $\begin{array}{c}0,34+ \\
0,004^{+}\end{array}$ & $\begin{array}{c}0,19+ \\
0,006\end{array}$ & $\begin{array}{c}0,18 \\
0,008\end{array}$ \\
\cline { 2 - 9 } & Signif. & $\mathrm{a}$ & $\mathrm{a}$ & $\mathrm{a}$ & $\mathrm{a}$ & $\mathrm{a}$ & $\mathrm{a}$ \\
\hline
\end{tabular}

1 mois

\begin{tabular}{|c|c|c|c|c|c|c|c|}
\hline \multirow{2}{*}{ Dulieu } & $\begin{array}{l}\text { Long. de } \\
\text { I'hypoc. }\end{array}$ & $\begin{array}{c}0,61 \\
0,13\end{array}+$ & $\begin{array}{c}0,62 \\
0,13\end{array}+$ & $\begin{array}{c}0,50 \\
0,10\end{array}+$ & $\begin{array}{c}0,36 \\
0,07\end{array}+$ & $\begin{array}{c}0,26 \\
0,05\end{array}+$ & $\underset{0,05}{0,23}+$ \\
\hline & Signif. & ef & ef & d & b & a & a \\
\hline \multirow{2}{*}{ xanthi } & $\begin{array}{l}\text { Long. de } \\
\text { l'hypoc. }\end{array}$ & $\begin{array}{c}0,590 \\
0,08\end{array}+$ & $\begin{array}{c}0,64 \\
0,049\end{array}+$ & $\begin{array}{c}0,56+ \\
0,048\end{array}$ & $\underset{0,12}{0,41}+$ & $\underset{0,14}{0,24}+$ & $\underset{0,12}{0,23}+$ \\
\hline & Signif. & ef & $f$ & e & c & a & a \\
\hline
\end{tabular}
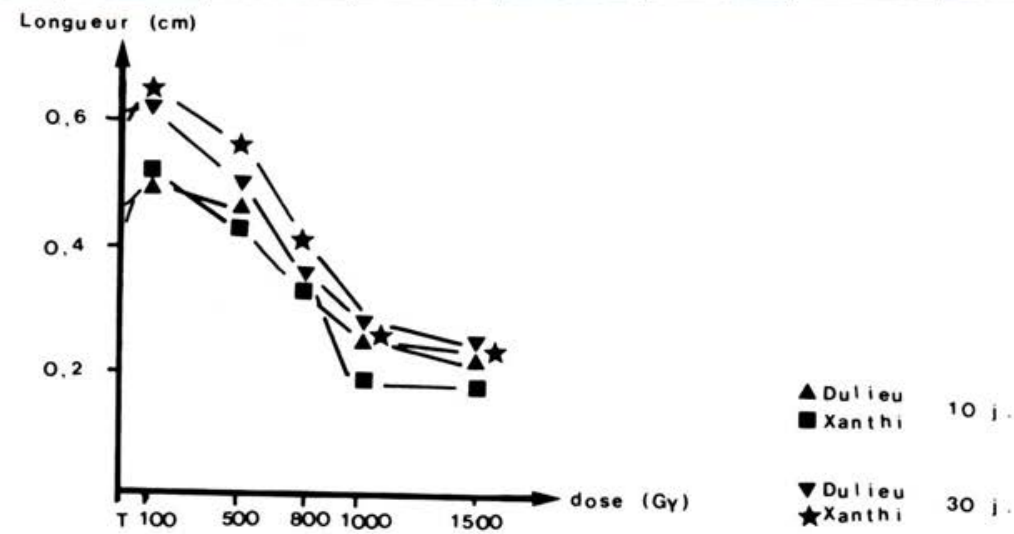

Fig. 7. - Influence de différentes doses de rayonnement $\gamma$ sur la longueur de l'hypocotyle mesurée chez deux variétés de tabac, 10 et 30 jours aprés le semis. 


\section{- Surface des cotylédons}

Dans les deux variétés, la surface des cotylédons des plantules issues de graines irradiées à 100 Gy est sensiblement équivalente à celle des témoins (légère augmentation de la surface non significative); les irradiations supérieures réduisent significativement le développement des cotylédons dont la surface pour 1500 Gy ne représente plus que $12 \%$ (var. xanthi) $28 \%$ (var. xanthi Dulieu) de celle des témoins. Notons que les cotylédons témoins de 10 jours sont à leur stade de maturité; par la suite, ils se déforment et flétrissent; en conséquence, nous n'avons pas mesuré leur surface au-delà de 10 jours après le semis (tableau VI et fig. 8).

\section{TABLEAU VI}

Influence de différentes doses de rayonnement $\gamma$ (en Gy) sur la surface de cotylédons mesurée chez deux variétés de tabac, 10 jours après le semis

\begin{tabular}{|c|c|c|c|c|c|c|c|}
\hline \multirow{4}{*}{ Dulieu } & Doses & Témoin & 100 & 500 & 800 & 1000 & 1500 \\
\cline { 2 - 8 } & $\%$ & $\begin{array}{c}6,33+ \\
0,07\end{array}$ & $\begin{array}{c}7,07+ \\
0,08\end{array}$ & $\begin{array}{c}4,63+ \\
0,16\end{array}$ & $\begin{array}{c}2,93+ \\
0,086\end{array}$ & $\begin{array}{c}1,87+ \\
0,03\end{array}$ & $\begin{array}{c}1,77+ \\
0,024\end{array}$ \\
\cline { 2 - 8 } & Signif. & $\mathrm{d}$ & $\mathrm{d}$ & $\mathrm{c}$ & $\mathrm{b}$ & $\mathrm{ab}$ & $\mathrm{ab}$ \\
\hline \multirow{4}{*}{ xanthi } & $\%$ & $\begin{array}{c}7,50+ \\
0,10^{+}\end{array}$ & $\begin{array}{c}7,71+ \\
0,33^{+}\end{array}$ & $\begin{array}{c}2,10+ \\
0,10^{+}\end{array}$ & $\begin{array}{c}1,20+ \\
0,04\end{array}$ & $\begin{array}{c}1,20+ \\
0,035^{+}\end{array}$ & $\begin{array}{c}0,90+ \\
0,02\end{array}$ \\
\cline { 2 - 8 } & Signif. & $\mathrm{d}$ & $\mathrm{d}$ & $\mathrm{ab}$ & $\mathrm{a}$ & $\mathrm{a}$ & $\mathrm{a}$ \\
\hline
\end{tabular}

Surtace $\left(\mathrm{mm}^{2}\right)$

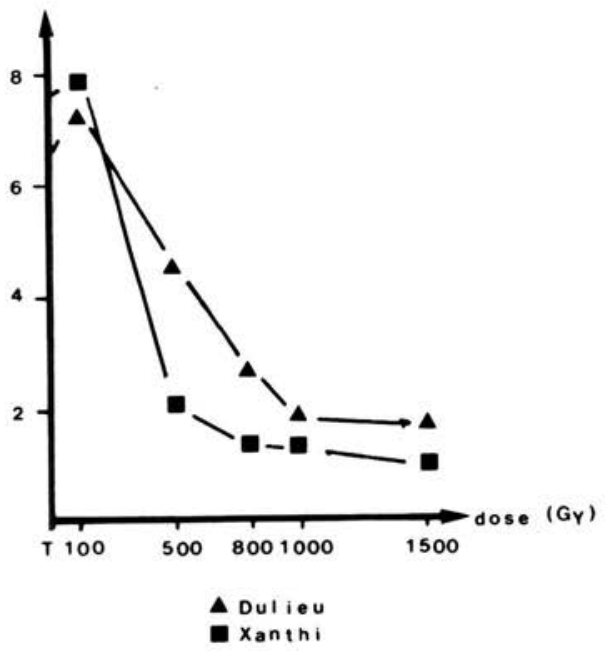

Fig. 8. - Influence de différentes doses de rayonnement $\gamma$ sur la surface de cotylédons mesurée chez deux variétés de tabac, 10 Jours après le semis. 


\section{III.3.2. Observation 30 Jours après le semis (ou irradiation)}

Entre le $10^{\circ}$ et le $30^{e}$ jour après le semis, la plantule témoin a vu de nombreuses racines latérales se développer, l'hypocotyle s'est faiblement allongé (de $0,45 \mathrm{~cm}$ à $0,60 \mathrm{~cm}$ ) et l'axe épicotyle (tige) commence à se développer. Au $30^{\mathrm{e}}$ jour, on note la présence des 3 premières feuilles.

\section{- Longueur de la racine principale}

Entre le $10^{e}$ et le $30^{\circ}$ jour de culture, les racines principales des plantules issues des graines irradiées continuent de s'allonger (tableau VIlb et fig. 6). Les longueurs des racines principales chez les deux variétés sont maximales pour les plantules issues des graines irradiées à $100 \mathrm{~Gy}$, puis les valeurs décroissent quand les doses augmentent. Cependant, il est intéressant de noter qu'entre le $10^{\mathrm{e}}$ et le $30^{\mathrm{e}}$ jour, le faible accroissement de la longueur de la racine principale en valeur absolue chez la variété xanthi Dulieu est, en valeur relative à 1000 et $1500 \mathrm{~Gy}$, presque équivalent à celui des témoins (traités: $34,4 \%$, témoins: $39,4 \%$ ) et que cet accroissement relatif est optimal à 500 et 800 Gy respectivement pour les variétés xanthi Dulieu $(16,15 \%)$ et xanthi $(14,52 \%)$ pour un accroissement relatif du témoin de $4,98 \%$. Le développement radiculaire des plantules issues des graines irradiées à 1000 et 1500 Gy de la variété xanthi est insignifiant pendant la période considérée.

\section{TABLEAU VII}

Influence de différentes doses de rayonnement $\gamma$ (en Gy) sur la longueur de la racine mesurée chez deux variétés de tabac, 10 et 30 jours après le semis

10 jours

\begin{tabular}{|c|c|c|c|c|c|c|c|}
\hline \multirow{4}{*}{ Dulieu } & Doses & Témoin & 100 & 500 & 800 & 1000 & 1500 \\
\cline { 2 - 8 } & $\begin{array}{c}\text { Long. de } \\
\text { la rac. }\end{array}$ & $\begin{array}{c}0,87+ \\
0,18\end{array}$ & $\begin{array}{c}0,93+ \\
0,19\end{array}$ & $\begin{array}{c}0,20+ \\
0,04\end{array}$ & $\begin{array}{c}0,11+ \\
0,02\end{array}$ & $\begin{array}{c}0,09+ \\
0,019\end{array}$ & $\begin{array}{c}0,08+ \\
0,016\end{array}$ \\
\cline { 2 - 8 } & Signif. & $\mathrm{c}$ & $\mathrm{c}$ & $\mathrm{a}$ & $\mathrm{a}$ & $\mathrm{a}$ & $\mathrm{a}$ \\
\hline \multirow{3}{*}{ xanthi } & $\begin{array}{c}\text { Long. de } \\
\text { la rac. }\end{array}$ & $\begin{array}{c}0,89+ \\
0,18^{+}\end{array}$ & $\begin{array}{c}0,97+ \\
0,20^{+}\end{array}$ & $\begin{array}{c}0,44^{+} \\
0,09\end{array}$ & $\begin{array}{c}0,23+ \\
0,05\end{array}$ & $\begin{array}{c}0,11+ \\
0,02\end{array}$ & $\begin{array}{c}0,11+ \\
0,022\end{array}$ \\
\cline { 2 - 8 } & Signif. & $\mathrm{c}$ & $\mathrm{c}$ & $\mathrm{b}$ & $\mathrm{a}$ & $\mathrm{a}$ & $\mathrm{a}$ \\
\hline
\end{tabular}

1 mois

\begin{tabular}{|c|c|c|c|c|c|c|c|}
\hline \multirow{4}{*}{ Dulieu } & $\begin{array}{c}\text { Long. de } \\
\text { la rac. }\end{array}$ & $\begin{array}{c}3,93+ \\
0,18\end{array}$ & $\begin{array}{c}4,60+ \\
0,08\end{array}$ & $\begin{array}{c}3,43+ \\
0,08\end{array}$ & $\begin{array}{c}1,43+ \\
0,10^{+}\end{array}$ & $\begin{array}{c}0,40+ \\
0,02\end{array}$ & $\begin{array}{c}0,30+ \\
0\end{array}$ \\
\cline { 2 - 8 } & Signif. & cde & efg & c & b & a & a \\
\hline \multirow{3}{*}{ xanthi } & $\begin{array}{c}\text { Long. de } \\
\text { la rac. }\end{array}$ & $\begin{array}{c}5,33+ \\
0,07\end{array}$ & $\begin{array}{c}5,97+ \\
0,11^{+}\end{array}$ & $\begin{array}{c}4,30+ \\
0,06\end{array}$ & $\begin{array}{c}3,57+ \\
0,1\end{array}$ & $\begin{array}{c}0,20+02 \\
0,02\end{array}$ & $\begin{array}{c}0,13+ \\
0,01\end{array}$ \\
\cline { 2 - 8 } & Signif. & gh & $\mathrm{h}$ & def & cd & a & a \\
\hline
\end{tabular}




\section{- Longueur de l'hypocotyle}

Chez les deux variétés (tableau $\mathrm{Vb}$ et fig. 7 ), la majeure partie (75\% environ) de l'élongation de l'hypocotyle des témoins s'effectue au cours des 10 premiers jours de la culture; il en va de même ( $82 \%$ enviroñ) pour les hypocotyles issus des graines irradiées à 100 Gy qui, dans les deux variétés, entre le $10^{\mathrm{e}}$ et le $30^{\mathrm{e}}$ jour, s'allongent proportionnellement moins que ceux des témoins: 21,5 et $23 \%$ à 100 Gy contre 33 et $37 \%$ chez les témoins. A $500 \mathrm{~Gy}$, l'élongation est encore importante entre le $10^{\mathrm{e}}$ et le $30^{\mathrm{e}}$ jour chez la variété xanthi, elle est faible dans la variété xanthi Dulieu. Au-delà, l'élongation est faible partout.

\section{- Longueur de la tige}

Au $10^{\mathrm{e}}$ jour de culture (tableau VIII et fig. 9), les axes épicotylés des plantules témoins des deux variétés n'ont pas encore amorcé leur développement en tiges feuillées mais, après 30 jours de culture, ces tiges mesurent

\section{TABLEAU VIII}

Influence de différentes doses de rayonnement $\gamma$ (en Gy) sur la longueur de la tige mesurée chez deux variétés de tabac, 30 jours après le semis

\begin{tabular}{|c|c|c|c|c|c|c|c|}
\hline \multirow{4}{*}{ Dulieu } & Doses & Témoin & 100 & 500 & 800 & 1000 & 1500 \\
\cline { 2 - 8 } & $\begin{array}{c}\text { Long. de } \\
\text { la tige }\end{array}$ & $\begin{array}{c}2,30+ \\
0,1\end{array}$ & $\begin{array}{c}2,50+ \\
0,08\end{array}$ & $\begin{array}{c}2,06+ \\
0,07\end{array}$ & $\begin{array}{c}1,33+ \\
0,05\end{array}$ & $\begin{array}{c}0,57+ \\
0,07\end{array}$ & \\
\cline { 2 - 8 } & Signif. & cd & cde & cde & b & a & X \\
\hline \multirow{4}{*}{ xanthi } & $\begin{array}{c}\text { Long. de } \\
\text { la tige }\end{array}$ & $\begin{array}{c}2,43+ \\
0,08\end{array}$ & $\begin{array}{c}2,90+ \\
0,02\end{array}$ & $\begin{array}{c}2,09+ \\
0,01\end{array}$ & $\begin{array}{c}1,50+ \\
0,02\end{array}$ & $\begin{array}{c}0,13+ \\
0,01\end{array}$ & \\
\cline { 2 - 8 } & Signif. & cde & de & e & c & a & X \\
\hline
\end{tabular}

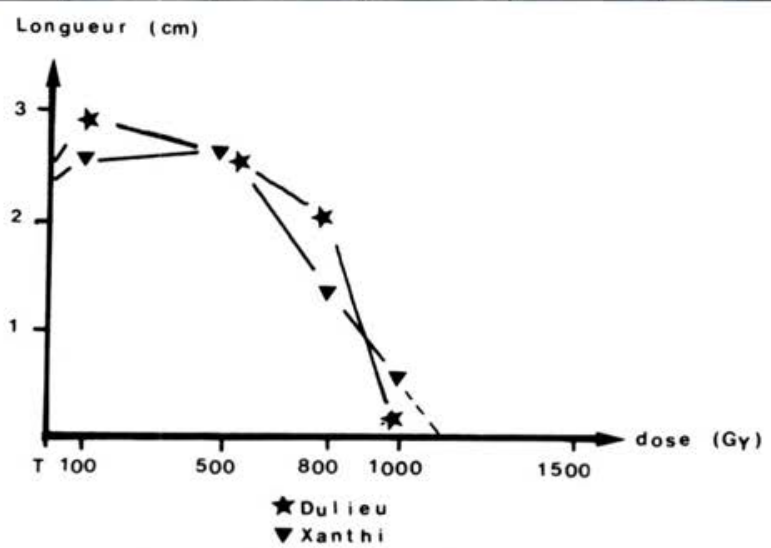

Fig. 9. - Influence de différentes doses de rayonnement $\gamma$ sur la longueur de la tige mesurée chez deux variétés de tabac, 10 et 30 jours après le semis. 
$2,30 \mathrm{~cm}$ (var. xanthi Dulieu) et $3,43 \mathrm{~cm}$ (var. xanthi). L'irradiation à $100 \mathrm{~Gy}$ des graines induit un développement légèrement supérieur des tiges pour la même période de végétation $(+0,20 \mathrm{~cm}$ : var. xanthi Dulieu; $+0,47 \mathrm{~cm}$ : var. xanthi) mais la différence mesurée n'est pas significative. Pour des irradiations de 500 et 800 Gy, le développement de l'axe caulinaire est au contraire nettement réduit: à 1000 Gy les plantules survivantes - la majorité des plantules issues de graines irradiées à 1000 Gy meurent entre le $10^{\mathrm{e}}$ et le $30^{\mathrm{e}}$ jour après le semis - ne forment que de très petites tiges correspondant au 1/4 (var. xanthi Dulieu) ou au 1/18 (var. xanthi) de celles des plantes témoins. Le développement caulinaire de la variété xanthi est plus stimulé par l'irradiation à 100 Gy que celui de la variété xanthi Dulieu, cette dernière se montrant par contre plus résistante aux irradiations supérieures. Une irradiation de 1500 Gy des graines entraîne une inhibition complète du développement des plantules au-delà du stade des deux cotylédons étalés et une mortalité de $100 \%$ de ces plantules avant le $30^{\mathrm{e}}$ jour.

\section{DISCUSSION}

La détermination de la radiosensibilité des graines des deux variétés de tabac à partir des tests biologiques de germination révèle une résistance remarquable du tabac à l'irradiation dans la mesure où même la dose la plus élevée (1500 Gy en 7 h 30 min d'exposition) n'inhibe pas totalement les processus physiologiques permettant le développement d'embryons jusqu'au stade jeunes plantules à cotylédons déployés.

Une observation détaillée (non publiée) des différentes phases de la germination des graines irradiées montre que (i) plus la dose employée est importante, plus le blocage de germination, lorsqu'il existe, est précoce, (ii) la phase initiale de germination (phase I: éclatement des téguments et sortie de la radicule) des graines traitées est faiblement inhibée par rapport à l'inhibition naturelle des témoins, ce qui prouve la résistance des embryons aux doses employées et corrobore les observations de DUBININ [9].

Selin ILIEVA et MOLKHOVA [17], chez Capsicum annuum et Pendulinum, l'irradiation bloquerait le développement de l'embryon et entraînerait sa dégénérescence; les auteurs relient ces phénomènes à un arrêt de la division cellulaire dans l'albumen qui dégénère également.

Deux phénomènes majeurs relatifs à la morphologie des plantules de tabac issues de graines soumises à différentes doses de rayonnement se dégagent de nos observations:

- Une stimulation de croissance à la faible dose du gradient choisi (100 Gy). Bien que non significative au seuil de $5 \%$, elle rejoint les observations de SHEPPARD et EVENDEM (Triticum aestivum) et de SPARROW et EVANS (Vicia faba et Lilium IOngiflorum) [24, 26].

L'observation de MALTSEVA [20] chez la tomate, prouvant que, plus la teneur en eau des graines est basse, plus l'effet stimulant des faibles doses de rayonnement se fait sentir, explique peut-être la stimulation observée chez les graines de tabac faiblement hydratées. MOSNOVA et MALTSEVA [21] 
relient l'effet stimulant des faibles doses à une activation de la polyphénoloxydase dans les graines soumises à l'irradiation et dans les plantules issues de ces graines.

- Une inhibition du développement aux plus fortes doses du gradient, qui se caractérise essentiellement par (i) un accroissement plus réduit du système radiculaire, (ii) une élongation plus faible de l'hypocotyle, (iii) une réduction de la surface cotylédonaire, (iv) une longueur de la tige mesurée après 30 jours inférieure à celle des témoins. Ces observations vont dans le même sens que celles de CALLEBAUT et al. effectuées sur l'axe du pois, de SOLER [25] chez le riz, de CONGER et STEVENSON [2] chez l'orge et de DATTA et al. [1], chez Nigella sativa L.; notons que CONGER et STEVENSON et DATTA et al. relient la diminution de la taille des jeunes plants à une augmentation des altérations nucléaires dans les tissus des plantules.

Exception faite de la stimulation à 100 Gy de l'allongement caulinaire, les différences morphologiques, même ténues, observées entre les deux variétés indiquent, à l'image des observations relatives à la germination, que la variété xanthi Dulieu résiste mieux aux effets nocifs du rayonnement.

\section{CONCLUSION}

Les résultats montrent que (i) la radiorésistance des graines des deux variétés est grande, (ii) sauf exception (pourcentage des plantules ayant formé deux cotylédons significativement plus faible dans la variété Xanthi), la différence de radiosensibilité entre les deux variétés n'est pas très grande; notons toutefois que la majorité des résultats significatifs présentent un déplacement des valeurs en faveur d'une plus grande radiorésistance de la variété Xanthi Dulieu, (iii) la forme de la réponse dose-effet est du même type quels que soient la variété et le critère biologique retenus:

- stimulation à la faible dose du gradient choisi,

- inhibition de la germination et du développement aux plus fortes doses (1000 et 1500 Gy).

\section{REMERCIEMENTS}

Nous remercions vivement:

- M. L. ALBERTINI, professeur à I'INPT-ENSAT pour ses conseils;

- M. A. SOUVRE, maître de conférences (habilité à diriger des recherches) à I'INPTENSAT pour ses conseils et sa participation à la rédaction de cet article;

- MIle S. ALAMI pour sa participation à la rédaction de cet article;

$-M_{\text {. H. }}$. DULIEU, qui nous a fourni les graines de la variété portant le système $\underline{a}_{1}^{+} \underline{a}_{1}$ $\underline{a}_{2}^{+} / \underline{a}_{2}$.

- MM. les responsables de l'Institut expérimental du tabac de Bergerac qui nous ont fourni les graines de la variété xanthi.

- MM. les professeurs MATHIEU et TORRES de I'ENSC de Toulouse, qui ont mis la "gamma cell" à notre disposition. 


\section{RÉFÉRENCES BIBLIOGRAPHIQUES}

[1] DATTA A.K., BISWAS A.K., SEN. S. Gamma radiation sensitivity in Nigella sativa L. Cytologia, 51, 1986, 609-615.

[2] CONGER A.D., STEVENSON H.Q. A correlation of seedling height and chromosomal damage in irradiated barley seeds. Radiat. Bot., 1969, 9, 1-14.

[3] DELPOUX M., DALEBROUX M. Genetic effects on the $\underline{a}_{1}^{+} / \underline{a}_{1} \underline{a}_{2}^{+} / a_{2}$ system of tobacco over a uranous outcrop in the Permian Basin of Lodève (Hérault, France). Mutat. Res., 1981, 82, 101-110.

[4] DELPOUX M., DALEBROUX $M$. Genetic effects on the $\underline{a}_{1}^{+} / \underline{a}_{1} \underline{a}_{2}^{+} / \underline{a}_{2}$ system of tobacco over a uranous outcrop in Lauragais (Aude, France). Mutat. Res., 1981, $83,375-382$.

[5] DELPOUX M., DALEBROUX M. Effets génétiques de sols du Pays de Sault (Aude), différant par leur radioactivité et leur composition chimique. Comptes rendus du Congrès national des sociétés savantes, Perpignan. Sciences, 1981, II, 233-244.

[6] DEVAUD B. Evaluation in situ des effets génétiques d'atmosphères diversement polluées de l'agglomération de Toulouse (France). Thèse $3^{e}$ cycle, Université P.Sabatier, Toulouse, 1986.

[7] DEVAUD B., DELPOUX M., DALEBROUX M. Evaluation in situ des effets génétiques induits par des atmosphères diversement polluées de l'agglomération toulousaine. In: Comptes rendus du Symposium international d'écotoxicologie, Les Arcs, 1984. Paris: ministère de l'Environnement, 183-197.

[8] DEVAUD B., DELPOUX M., DALEBROUX M. Expérimentation en enceintes fermées à air filtré et non filtré pour évaluer in situ les effets génétiques de la pollution atmosphérique. In: Proceeding of the Clean air congress, Sydney, 1986. International union of air pollution prevention associations, 4, 140-147.

[9] DUBININ N.P. Problems of radiations genetics. Edinburgh: Oliver and Boyd, 1961, 163-187.

[10] DULIEU H. Etude de la stabilité d'une déficience chlorophyllienne induite par traitement au méthane sulfonate d'éthyle. Ann. Amélior. Plantes, 1967, 17 (1) 339-355.

[11] DULIEU $H$. Somatic variations on a yellow mutant in Nicotiana tabacum $L$. $\left(a_{1}^{+} / a_{1} \underline{a}_{2}^{+} / a_{2}\right)$. 1. Non-reciprocal genetic events occurring in leaf cells. Mutat. Res., $1974,25,289-304$.

[12] DULIEU H. Somatic variations on a yellow mutant in Nicotiana tabacum L. $\left(\mathrm{a}_{1}^{+} / \mathrm{a}_{1} \mathrm{a}_{2}^{+} / \mathrm{a}_{2}\right)$. 2. Reciprocal genetic events occurring in leaf cells. Mutat. Res., $1975,28,66-77$.

[13] DULIEU $\mathrm{H}$. Antimorphic alleles with various strengths at the $\mathrm{Su}$ and $\mathrm{Yg}$ loci of tobacco. J. Heredity, 1986, 77 (5) 301-306.

[14] DULIEU H., DALEBROUX M. Spontaneous and induced rates in a double heterozygous mutant of Nicotiana tabacum var. Xanthi n.c.; dose-response relationship. Mutat. Res., 1975, 30, 63-70.

[15] FABRIES M., DELPOUX M. Genetic effects of low and very low chronic doses of gamma irradiation on the $\underline{a}_{1}^{+} / \underline{a}_{1} \underline{a}_{2}^{+} / \underline{a}_{2}$ system of tobacco. Mutat. Res., 1978,49 , 377-382.

[16] GHOSH N., SEN S. Assessment of radiosensitivity from germination of jute seeds. Biologia Plantarum (Praha), 1978, 20 (4) 268-273. 
[17] ILIEVA I., MOLKHOVA E. Etude du développement de l'embryon et de l'albumen chez Capsicum annum et C. pendulum, après irradiation. Genet. Sclek., Bâlg., 1976, 9 (5) 400-404.

[18] KIVI E.I. On sterility and other injuries in dioccions Melondrium irradiated with $\mathrm{X}$ rays and gamma rays. Ann. Acad. Sci., Fenn. AIV, 1962, 56, 7-96.

[19] LINSHAIER E., SKOOG F. Organic growth factor requirements of tobacco tissue cultures. Physiol. Plant., 1965, 18, 100-127.

[20] MALTSEVA S. Influence de la teneur en eau des graines de tomate sur l'effet stimulant dû aux faibles doses de rayons $\gamma$ du cobalt 60. Radiobiologiya SSSR, 1977, 17 (1) 138-141.

[21] MOSNOVA K., MALTSEVA S. Influence des radiations ionisantes sur le développement des plantes et sur l'activité de la polyphénol-oxydase. Radiobiologiya SSSR, 1978, 18 (5) 774-777.

[22] MURASHIGE T., SKOOG F. A revised medium for rapid growth and bioassays with tobacco tissue cultures. Physiol. Plant., 1962, 15, 473-497.

[23] Semences et Progrès, 1975, $n^{\circ} 6$.

[24] SHEPPARD S.C., EVENDEN W.G. Factors controlling the response of field crops to very low doses of gamma irradiation of the seed. Conference on Radiation hormesis, Oakland, Aug. 14-16, 1985. Can. J. Plant. Sci., 1986, 66, 431.

[25] SOLER A. Définition de conditions d'irradiation et de traitement susceptibles de réduire sur des semences de riz les effets physiologiques du rayonnement gamma du cobalt 60 . Thèse $3^{e}$ cycle, Montpellier, 1974, p. 203.

[26] SPARROW A.H., EVANS H.J. Nuclear factors affecting radiosensitivity. 2. The influence of nuclear size and structure, chromosome complement and DNA content. Brookhaven Symp. Biol., 1961, 14 (1) 76-100. 